\title{
La competencia digital de los estudiantes universitarios de primer curso de grado
}

\section{First-year College Students' Digital Competence}

\author{
Anna Sánchez-Caballée ${ }^{1}$, Mercè Gisbert-Cervera ${ }^{2}$, Francesc Esteve-Mon ${ }^{3}$ \\ ${ }^{1}$ Universitat Rovira i Virgili, España (anna.sanchez@urv.cat) \\ ${ }^{2}$ Universitat Rovira i Virgili, España (merce.gisbert@urv.cat) \\ ${ }^{3}$ Universitat Jaume I, España (festeve@uji.es)
}

Recibido el 8 de febrero de 2019; revisado el 3 de abril de 2019; aceptado el 3 de abril de 2019; publicado el 1 de diciembre de 2019

\section{RESUMEN:}

Aunque el discurso sobre competencia digital no es nuevo, la investigación sobre el concepto y su medición siguen siendo una temática de actualidad. En esta línea el presente artículo evalúa la competencia digital de 168 estudiantes de primer curso de educación (Educación Infantil, Educación Primaria, doble titulación, Educación Social y Pedagogía) de la Facultad de Educación y Pedagogía de la Universitat Rovira i Virgili mediante el cuestionario de autopercepción INCOTIC durante el curso académico 2017-2018. Este tipo de cuestionarios cobran importancia en referencia a la adaptación y la perspectiva de constante evolución, ya que permiten reconocer las dificultades y potencialidades de los estudiantes (DomínguezAlfonso, Hernández-Mendo y Chica-Merino, 2018). Los resultados muestran que estos estudiantes disponen de fácil acceso a la tecnología, pero no hacen mucho uso de esta. Contrariamente a lo que se puede esperar, aunque hacen poco uso de esta, se muestran muy predispuestos para la integración de la tecnología en sus procesos de trabajo. Finalmente, en referencia a su nivel de competencia digital, se puede considerar que estos tienen una autopercepción alta, especialmente de esas habilidades relacionadas con la comunicación y los elementos multimedia que son las que más utilizan en su vida diaria. Los resultados obtenidos con el proceso son útiles para mejorar la formación de estos estudiantes de grado y poder reformular, en caso de ser necesario, los planes de estudio.

\section{PALABRAS CLAVE: COMPETENCIA DIGITAL, ALFABETIZACIÓN DIGITAL, UNIVERSIDAD, EDUCACIÓN SUPERIOR.}

\begin{abstract}
:
Although the discourse on digital competence is not new, research on the concept and its measurement remain a current issue. According to that idea, this article evaluates the digital competence of 168 firstyear students in the Bachelor of Education programme (Preschool Education, Primary Education, double Bachelor's Degree in Preschool and Primary Education, Social Education and Pedagogy) in the Faculty of Education and Pedagogy at the Universitat Rovira i Virgili by means of the use of INCOTIC (a self-perception questionnaire) during the academic year 2017-2018. The results show that these students can easily access technology but do not make much use of it. However, although they make limited use of technology, they are very predisposed to integrate it in their work. Finally, in reference to their digital competence level, they have a high self-perception, especially in those skills related to communication and multimedia elements, which are the ones that they most commonly use in their everyday life. The results obtained with the research could be useful to improve the ICT training of these students and the abovementioned Bachelors' study plans.
\end{abstract}


KEYWORDS: DIGITAL COMPETENCE; DIGITAL LITERACY; UNIVERSITY; HIGHER EDUCATION.

\section{INTRODUCCIÓN}

La sociedad de la información y el conocimiento (SIC) y las nuevas demandas derivadas de esta han incrementado el interés a nivel internacional por modificar los planes educativos y adecuarlos al nuevo contexto, lo cual implica una nueva concepción curricular y un replanteamiento de los procesos de aprendizaje implicados (Bates, 2015; Mishra y Kereluik, 2011). En esta línea, diferentes instituciones han puesto el énfasis, en los últimos años, en las denominadas competencias clave o key competences, de las cuales, una de las más reiteradas en dichos modelos es la competencia digital (Gisbert et al., 2016). Según la Comisión Europea (2018), la competencia digital (en adelante, CD) implica el uso seguro, crítico y responsable de las tecnologías digitales, ya sea en el aprendizaje, el trabajo o la participación en la sociedad, e incluye una serie de alfabetizaciones en diferentes áreas, que van desde la información y los datos, la comunicación o la creación de contenidos, entre otros aspectos.

Respecto a la CD diversos autores consideran que hay que disponer de un buen nivel para poder desarrollarse de un modo exitoso, y que la universidad debe garantizar oportunidades para su adquisición (Gisbert, González y Esteve, 2016). Como indica la OCDE (2018), los estudiantes actuales tendrán que aplicar sus conocimientos en contextos digitales muy cambiantes y circunstancias desconocidas actualmente, por lo que será necesaria una formación digital (Erstad, 2010).

Sin embargo, las prácticas realizadas en la universidad española no acaban de dar respuesta a la CD requerida (Guzmán-Simón, García-Jiménez y López-Cobo, 2017). Por otro lado, a pesar de que en las últimas décadas se hayan popularizado términos como nativo digital o similares (Prensky, 2001; Tapscott, 1998), diferentes autores han puesto de manifiesto que, pese a haber crecido en un contexto digital, esta generación no posee un nivel óptimo de $\mathrm{CD}$, sino que disponen de ciertas habilidades más desarrolladas, especialmente relacionadas con actividades de ocio y socialización, pero con muy poca transferencia a contextos académicos y laborales (Bullen, Morgan, Belfer, y Qayyum, 2011; Gallardo-Echenique, Marqués-Molías, Bullen y Strijbo, 2015; Kennedy et al., 2009).

Partiendo de estas premisas, la presente investigación pretende profundizar en el estudio de la CD de los estudiantes universitarios. En concreto, el propósito de este artículo es evaluar, a partir de su autopercepeción, la CD de los estudiantes de primer curso de los grados de educación teniendo en cuenta factores como el uso, el acceso, las expectativas y las actitudes que tienen hacia las TIC. Cabe destacar que, aunque el concepto de alfabetización digital suele ser el más utilizado a en el mundo anglosajón, en el contexto europeo se utiliza, de manera sinónima, el término CD (Ferrari, 2012), nomenclatura que se utilizará en el presente estudio.

\section{ANTECEDENTES Y FUNDAMENTOS TEÓRICOS}

\section{$2.1 \quad$ Uso de las TIC}

En las últimas décadas, las tecnologías de la información y la comunicación (en adelante TIC) se han incorporado de un modo notable en la vida cotidiana de los ciudadanos. Como muestra de esta realidad, el INE (2018) indica que el $86.4 \%$ de los hogares españoles dispone de conexión a internet, el $72.1 \%$ hace un uso de internet a diario, o que el $43.5 \%$ de las personas de entre 16 y 74 años han comprado por internet en los últimos tres meses, datos que van progresivamente creciendo año a año. Según la Asociación para la Investigación en Medios (AIMC) el acceso a internet se realiza mediante del teléfono móvil por parte de un $92.1 \%$ de la población y mediante el ordenador portátil por un $77.5 \%$. En referencia a la frecuencia de uso la mayor parte de ellos se encuentran en un uso constante o de varias veces al día, concretamente el $54.4 \%$ corresponde al primer grupo y el $38.9 \%$ al segundo.

Pero este avance no solamente se produce en el ámbito individual, sino que también es un hecho que se puede observar en el contexto educativo, y en concreto en educación superior. Tal y como indica Gómez (2017), el 83\% de las aulas universitarias está equipada con wifi y proyector y el $91 \%$ de los estudiantes se conectan, en algún momento, a la red wifi de la universidad.

Carbonell, Chamarro, Oberst, Rodrigo y Prades (2018) mantienen que en la última década se ha extendido el uso de internet y añaden que, junto con ello, también ha prosperado un consumo problemático, tanto de la red, como de los distintos dispositivos con pantallas, especialmente los móviles. Aun así, la concepción problemática no es extendida a todas las acciones que se realizan, sino que corresponde a un uso específico, es decir, que depende de la actividad que se lleva a cabo. En 2018, Kuss, Kanjo, Crook-Rumsey, Kibowski, Wang y Sumich apuntaban que el uso adictivo de los 
móviles solía ser proporcional al nivel de estrés del usuario, siendo los más afectados quienes hacían un mayor uso de estos.

Pero, si se mira más allá, autores como VegaHernández, Patino-Alonso y Galindo-Villardón (2018) afirman que los estudiantes universitarios realizan un uso básico y personal de la tecnología correspondiente a herramientas de comunicación, portales de vídeo y buscadores web. Aún así, los estudiantes consideran que las TIC constituyen una importante ayuda en el proceso de aprendizaje y que es necesario una innovación educativa, desde la perspectiva de la incorporación de la tecnología. No obstante, es importante remarcar que un factor clave para que los estudiantes vivan positivamente y con buena actitud la incorporación de la tecnología en su ámbito profesional está relacionado con la percepción que tengan de su utilidad y de la facilidad de uso (Edmunds, Thorpe y Conole, 2012).

\subsection{Actitudes y expectativas hacia las TIC}

Las actitudes que tienen los estudiantes hacia el uso de las TIC suelen ser positivas, especialmente si, como se ha indicado en el punto anterior son útiles para las tareas que se van a realizar y fáciles de usar (Edmunds, Thorpe y Conole, 2012). Un ejemplo de ello son los estudiantes participantes en la investigación de Espuny, González, Lleixà y Gisbert (2011) quienes no habían utilizado previamente las redes sociales en el campo académico $\mathrm{y}$, sin embargo, presentaron una buena actitud hacia ellas. Cabe subrayar que algunos de estos estudiantes ya habían hecho uso de ellas en su vida cotidiana y tenían un buen dominio.

Por otro lado, cabe destacar que. en cierta parte, la actitud hacia la incorporación de las TIC depende de la dificultad del contenido y la ansiedad que este puede proporcionar a los estudiantes (Erdener y Kandemir, 2019).

Además, es necesario destacar que las expectativas que tienen los estudiantes cuando se habla de la integración de las TIC y, más concretamente con internet, viene en parte afectada con las experiencias previas y la autoeficiencia en el uso de las TIC. Las experiencias previas también están relacionadas muchas veces con el contexto en el que creció el alumno, hecho que afecta directamente a las habilidades que presenta (Hatlevik, Throndsen, Loi y Gudmundsdottir, 2018).

\subsection{Alfabetizaciones y Competencia Digital}

Gilster (1997) fue uno de los primeros autores que habló de la alfabetización digital considerándola como la capacidad de un estudiante para el acceso, la evaluación y la gestión de la información multimodal. Este término, alfabetización o $\mathrm{CD}$, así como su definición, ha ido variando y evolucionando dependiendo del momento temporal y el autor. Con la llegada de la tecnología, un estudiante estaba alfabetizado digitalmente cuando disponía de diversas habilidades relacionadas con el acceso, la evaluación y la gestión de la información, es decir, era capaz de construir conocimiento utilizando diversas fuentes y sabiendo determinar la veracidad de estas (Ferrari, 2012). Además, hay que tener en cuenta el valor tanto de las herramientas tradicionales como de las actuales creando redes de colaboración y cooperación para publicar y comunicar información (Bawden, 2008; Nawaz y Kundi, 2010). Moreno y Delgado en 2013 destacaron la importancia para los estudiantes de disponer de una adecuada CD. En esa misma línea, diversos autores $\mathrm{e}$ instituciones han elaborado diferentes marcos y modelos para tratar de conceptualizar dicha competencia. A continuación, destacamos algunos de ellos:

- Eshet-Alkalai (2004). Propone un modelo conceptual de alfabetización digital holístico que incluye las alfabetizaciones: (1) foto-visual, el arte de leer representaciones visuales; (2) reproductiva, el arte de reciclar creativamente los materiales existentes; (3) ramificada, hipermedia y pensamiento no lineal; (4) informacional, el arte del escepticismo y (5) socio-emocional.

- ISTE (2007). La International Society for Technology in Education (ISTE) propone un marco de CD que se desarrolla a partir de varios estándares llamados National Educational Technology Standards (NETS). Los estándares corresponden a: (1) creatividad e innovación; (2) comunicación y colaboración; (3) búsqueda y afluencia de información; (4) pensamiento crítico, resolución de problemas y toma de decisiones; (5) ciudadanía digital y (6) conceptos y acciones tecnológicas.

- Mozilla (2015). Es el marco que propone Mozilla como guía para ejercer la buena ciudadanía web. Para ello cada usuario debe tener cuatro habilidades correspondientes a la era digital: (1) lectura; (2) escritura; (3) participación y (4) las 21 century skills que corresponden al conjunto de conocimientos, habilidades y hábitos de trabajo necesarios para tener éxito en el mundo de hoy.

- Carretero, Vuorikari y Punie (2017). El modelo propuesto por el Institute for Prospective Technological Studies (IPTS), Digcomp 2.1 es la continuación actualizada del Digcomp 2.0 (Vuorikari, Punie, Carretero, Brande, 2016) y el 
Digcomp (Ferrari, 2012). Este modelo considera cinco áreas: (1) alfabetización relacionada con la información y los datos, es decir, buscar, evaluar y gestionar la información y el contenido digital; (2) comunicación y colaboración mediante las tecnologías digitales, compartiendo contenidos, participando en la ciudadanía online siguiendo las normas de participación en red denominadas netiquette y gestionando la identidad digital; (3) creación de contenido digital, reelaborándolo, integrándolo y programando teniendo presente las licencias y los derechos de autor; (4) seguridad en referencia a la protección de los dispositivos, de los datos personales, la salud y el medio ambiente y (5) solución de problemas técnicos, identificación de necesidades y respuestas tecnológicas, uso creativo de la tecnología e identificación de problemas técnicos.

En su investigación doctoral, Larraz (2013), presenta un análisis panorámico de los diferentes marcos y modelos. La autora propone una visión aglutinadora del concepto de CD para el contexto universitario compuesta por cuatro alfabetizaciones, que unidas componen la CD: (1) informacional, es decir, saber hacer un manejo de la información; (2) tecnológica, referente al tratamiento de la información en diversos formatos digitalmente; (3) multimedia, correspondiente análisis y la creación de mensajes multimedia y (4) comunicativa, es decir, la participación, la comunicación mediante el uso de una identidad digital. Este concepto de $\mathrm{CD}$ es el que es tomado como referencia para la elaboración de INCOTIC (González, Esteve, Larraz, Espuny y Gisbert, 2018), una herramienta para la evaluación autopercibida de la CD de los estudiantes universtarios, que tomaremos como referencia en la presente investigación.

Más allá de las definiciones, si revisamos el perfil de los jóvenes universitarios autores como Mesároš VÚSI y Mesároš (2010) y Liesa Orús Vázquez-Toledo y Lloret-Gazo (2016) consideran que no tienen un alto nivel de $\mathrm{CD}$. El hecho de que hagan uso de las TIC no les hace competentes digitales. De hecho, sus habilidades son mayoritariamente técnicas y no tienen un nivel óptimo de habilidades informacionales y multimedia (Gabarda Méndez, Rodríguez Martín y Moreno Rodríguez, 2017; Ozdamar-keskin, Ozata, Banar y Royle, 2015).

En la misma línea, se considera que en numerosas ocasiones presentan dificultades para el uso de programas informáticos específicos para su ámbito de estudio y trabajo, como pueden ser el manejo de blogs y procesadores de texto (Roushan, Debbie y Biggins, 2016; Sharp, 2017; Simonics, 2017). En cambio, las habilidades propias de contextos informales sí que se encuentran más desarrolladas (Guzmán-Simón, García-Jiménez y López-Cobo, 2017). Por tanto, tal como señalan Gobel y Cano (2013) la cantidad de uso o el haber nacido en una generación concreta no define a los estudiantes como competentes a nivel digital, por lo que resulta necesario profundizar en un análisis amplio de su CD.

\section{MATERIAL Y MÉTODO}

La presente investigación se enmarca en un paradigma cualitativo ya que el grado de control sobre los participantes es inexistente, y bajo una metodología de dicha investigación descriptiva, a partir de un cuestionario autoadministrado con el que se obtienen datos sobre la CD de los estudiantes de primer curso de grados de educación de la Universitat Rovira i Virgili (URV).

El presente estudio se plantea las siguientes preguntas de investigación:

- P1. ¿Qué uso hacen los estudiantes universitarios de las tecnologías?

- P2. ¿Qué expectativas y actitudes tienen los estudiantes universitarios del uso de las tecnologías?

- P3. ¿Cuál es nivel de CD de los estudiantes universitarios según su autopercepción?

\subsection{Contexto de la investigación}

Los sujetos participantes han sido los estudiantes de primer curso de la Facultad de Educación y Psicología de la Universidad Rovira i Virgili, de los grados de Educación Infantil (EI), Educación Primaria (EP), doble Titulación de EI y EP (DT), Educación Social (ES) y Pedagogía (P). El muestreo escogido para la realización ha sido intencional $(\mathrm{N}=168)$, participando un $60 \%$ del total de plazas disponibles de primer curso.

Se ha elegido este colectivo con la intención de poder desarrollar una estrategia para el desarrollo de la CD a lo largo de los estudios de grado. A continuación, se presenta, de un modo detallado, la muestra de la que se dispone (tabla 1): 
Tabla 1. Características de la muestra

\begin{tabular}{lll}
\hline & & $\mathrm{n}(\%)$ \\
\hline Género & Hombre & $34(20.2)$ \\
& Mujer & $134(79.8)$ \\
\hline Grado & Educación Infantil & $46(27.4)$ \\
& Educación Primaria & $44(26.2)$ \\
& Doble titulación & $31(18.5)$ \\
& Educación Social & $27(16.1)$ \\
& Pedagogía & $20(11.9)$ \\
\hline Edad & $<18$ & $86(51.2)$ \\
& $19-20$ & $42(25)$ \\
& $>21$ & $40(23.8)$ \\
\hline
\end{tabular}

\subsection{Instrumento: INCOTIC, cuestionario de competencia digital}

El cuestionario INCOTIC consta de 104 ítems distribuidos en cinco partes del siguiente modo: (1) disponibilidad de recursos TIC (17 ítems repartidos en 6 bloques); (2) uso de los recursos TIC (39 ítems repartidos en 5 bloques); (3) CD (20 ítems distribuidos a partir de las alfabetizaciones propuestas por Larraz (2013): alfabetización informacional; alfabetización multimedia; alfabetización tecnológica; y alfabetización comunicativa); (4) actitudes (10 ítems); (5) expectativas (18 ítems).

El proceso de diseño de la herramienta y su validación se publicaron en González-Martínez, Esteve, Larraz, Espuny y Gisbert (2018). En este artículo se indica que, tras el pilotaje, los valores de correspondientes a la alfa de Cronbach corresponden a un 0.93 referente al apartado de CD y 0.91 en referencia al índice de expectativas y actitudes.

\section{RESULTADOS}

A continuación, se presentan los resultados obtenidos a partir de la aplicación del instrumento de autopercepción y presentamos los datos en tres apartados: (1) acceso y uso de las TIC; (2) expectativas y actitudes hacia las TIC y (3) CD. Este último ámbito, siguiendo a Larraz (2013), se ha analizado también a partir de las cuatro alfabetizaciones (multimedia, comunicacional, tecnológica e informacional). Además, cabe subrayar que la presentación de los datos varía según el origen de la información. Los datos de las distintas alfabetizaciones y de la CD se presentan en una escala sobre 5. En cambio, los datos sobre el acceso y el uso se explicitan en número de estudiantes y, también, en una escala sobre 100 .

La muestra utilizada tiene un margen de error inferior al 5\%, con una $\mathrm{N}=168$ estudiantes de una población total de 280 estudiantes.

\subsection{Acceso y uso de las TIC}

De los datos que nos han facilitado los estudiantes que han participado en la investigación podemos evidenciar que tienen fácil acceso a las TIC dado que el $97.6 \%$ dispone de un ordenador portátil e incluso el $100 \%$ de ellos tiene un teléfono móvil (tabla 2). Estos dos dispositivos, junto con la televisión, del que disponen un $97.6 \%$ de los estudiantes, son los más comunes entre ellos. A continuación, destacan las cámaras de fotos con un $79.2 \%$, las videoconsolas con un $66.7 \%$ y las tabletas. Finalmente, con un menor volumen de usuarios están los ordenadores de sobremesa de los que solamente disponen más o menos la mitad de los estudiantes, concretamente, el 52.4\%.

Tabla 2. Disponibilidad de recursos en el domicilio

\begin{tabular}{ccc}
\hline & \multicolumn{2}{c}{ TOTAL } \\
\hline Tipo de dispositivo & $\mathrm{n}(\%)$ & $\mathrm{n}(\%)$ \\
\hline Ordenador de sobremesa & $88(52.4)$ & $80(47.6)$ \\
Ordenador portátil & $164(97.6)$ & $4(2.4)$ \\
Tableta & $110(65.5)$ & $58(34.5)$ \\
Móvil & $168(100)$ & $0(0)$ \\
Televisión & $164(97.6)$ & $4(2.4)$ \\
Videoconsola & $112(66.7)$ & $56(33.3)$ \\
Cámara de fotos & $133(79.2)$ & $35(20.8)$
\end{tabular}

Algunos de los dispositivos presentados permiten el acceso a internet. Así pues, los estudiantes participantes disponen de conexión a internet en su domicilio de estudiantes en un $99.4 \%$ y un $98.2 \%$ en sus teléfonos móviles (tabla 3 ).

Tabla 3. Acceso a internet

\begin{tabular}{ccc}
\hline & \multicolumn{2}{c}{ TOTAL } \\
\hline & Sí & No \\
\hline Acceso a internet & $\mathrm{n}(\%)$ & $\mathrm{n}(\%)$ \\
\hline En el domicilio de estudiante & $167(99.4)$ & $1(0.6)$ \\
En el teléfono móvil & $165(98.2)$ & $3(1.8)$ \\
\hline
\end{tabular}

La conectividad en la Universidad se realiza principalmente mediante la conexión de la propia entidad educativa. El $94.6 \%$ de los estudiantes utiliza el wifi de la universidad y el $42.3 \%$ hace uso de su plan de datos móviles (tabla 4). 
Tabla 4. Acceso a internet en la Universidad

\begin{tabular}{ccc}
\hline & \multicolumn{2}{c}{ TOTAL } \\
\hline & Sí & No \\
\hline $\begin{array}{c}\text { Conexión en la } \\
\text { universidad }\end{array}$ & $\mathrm{n}(\%)$ & $\mathrm{n}(\%)$ \\
\hline $\begin{array}{c}\text { Wifi de la Universidad } \\
\text { Plan de datos móviles }\end{array}$ & $159(94.6)$ & $9(5.4)$ \\
\hline
\end{tabular}

En referencia al uso de dichos dispositivos para aprender en la Universidad se observa que el principal es el teléfono móvil con un $90.5 \%$ de los estudiantes utilizándolo con dicha finalidad, seguido de cerca por el ordenador portátil que es utilizado por el $85.7 \%$ de los estudiantes (tabla 5). Por otra parte, las opciones menos frecuentes son los equipos propios de la Universidad (50.6\%) y las tabletas, que son utilizadas por el $24.4 \%$ de los estudiantes.

Tabla 5. Recursos utilizados para aprender en la Universidad

\begin{tabular}{ccc}
\hline & \multicolumn{2}{c}{ TOTAL } \\
\hline Sé & No \\
\hline Recursos para aprender en la & $\mathrm{n}(\%)$ & $\mathrm{n}(\%)$ \\
Universidad & & \\
\hline Ordenador portátil & $144(85.7)$ & $24(14.3)$ \\
Tableta & $41(24.4)$ & $127(75.6)$ \\
Móvil & $152(90.5)$ & $16(9.5)$ \\
Equipos de la Universidad & $85(50.6)$ & $83(49.4)$ \\
\hline
\end{tabular}

Siguiendo con los recursos utilizados para el aprendizaje en el domicilio del estudiante, se observa la misma tendencia que en la Universidad. Los ordenadores portátiles y los móviles son los más utilizados, recibiendo una puntuación del $95.8 \%$ y $82.7 \%$ respectivamente (tabla 6). Las puntuaciones menores corresponden a las tabletas y los ordenadores de sobremesa, que están puntuados con un $34.5 \%$ y $32.1 \%$ respectivamente.

Tabla 6. Recursos utilizados para aprender en el domicilio

\begin{tabular}{ccc}
\hline & \multicolumn{2}{c}{ TOTAL } \\
\hline & Sí & No \\
\hline Recursos domicilio & $\mathrm{n}(\%)$ & $\mathrm{n}(\%)$ \\
\hline Ordenador de sobremesa & $54(32.1)$ & $114(67.9)$ \\
Ordenador portátil & $161(95.8)$ & $7(4.2)$ \\
Tableta & $58(34.5)$ & $110(65.5)$ \\
Móvil & $139(82.7)$ & $29(17.3)$ \\
\hline
\end{tabular}

Además de la disponibilidad también hay que hacer hincapié en el uso de dichos dispositivos. En la tabla 7 se puede observar que, mientras que de los dispositivos de sobremesa el $64.3 \%$ de los estudiantes no hacen nunca uso el $88.1 \%$ de ellos hace uso más de tres horas diarias de los dispositivos móviles, convirtiéndose así en los dispositivos más utilizados de todos. A ellos les siguen los portátiles, con un $31.5 \%$ de uso superior a 3 horas y un $34.5 \%$ entre 2 y 3 horas diarias.

Tabla 7. Horas diarias de uso de los dispositivos

\begin{tabular}{cccccc}
\hline \multicolumn{5}{c}{ TOTAL } & $\mathrm{n}(\%)$ \\
\hline & Nunca & $<1$ & $1-2 \mathrm{~h}$ & $2-3 \mathrm{~h}$ & $>3 \mathrm{~h}$ \\
& \multicolumn{5}{c}{ hora } \\
\hline Dispositivos & 108 & 33 & 14 & 7 & 6 \\
\hline Sobremesa & $(64.3)$ & $(19.6)$ & $(8.3)$ & $(4.2)$ & $(3.6)$ \\
Portátil & 4 & 13 & 40 & 58 & 53 \\
& $(2.4)$ & $(7.7)$ & $(23.8)$ & $(34.5)$ & $(31.5)$ \\
Tableta & 80 & 44 & 20 & 12 & 12 \\
& $(47.6)$ & $(26.2)$ & $(11.9)$ & $(7.1)$ & $(7.1)$ \\
Móvil & 1 & 3 & 7 & 9 & 148 \\
& $(0.6)$ & $(1.8)$ & $(4.2)$ & $(5.4)$ & $(88.1)$ \\
\hline
\end{tabular}

La puntuación media total del apartado de acceso y uso de las TIC por parte de los estudiantes recibe una puntuación de 2.42 sobre 5 , convirtiéndose en el apartado con una menor puntuación de los analizados (tabla 8).

Tabla 8. Resultados uso INCOTIC

\begin{tabular}{ccc}
\hline & Media & $\begin{array}{c}\text { Desviación } \\
\text { estándar }\end{array}$ \\
\hline Uso de las TIC & 2.42 & .55 \\
\hline
\end{tabular}

\subsection{Expectativas y actitudes hacia las TIC}

En referencia a los 10 ítems del cuestionario que corresponden a las expectativas en cuanto al uso de las TIC (tabla 9) se puede observar que la puntuación más alta, concretamente de 4.47, pertenece a la comunicación entre los compañeros. Cerca de dicho ítem, con una puntuación de 4.31, está el ítem propio del ámbito docente, es decir, ese que hace referencia al agrado de los estudiantes por el uso de las TIC por parte del profesorado. En el lado contrario, es decir, la puntuación más baja, se encuentra la escritura, es decir, el referente a la ayuda que proporcionan las TIC para la mejora de su escritura que concretamente se puntúa con un 3.72. 
Tabla 9. Expectativas hacia las TIC

\begin{tabular}{lcc}
\hline & Media & $\begin{array}{c}\text { Desviación } \\
\text { estándar }\end{array}$ \\
\hline 1. Facilidad de uso & 4.24 & .80 \\
2. Sensación de disfrute & 4.23 & .80 \\
3. Autonomía & 4.27 & .77 \\
4. Comunicación & 4.47 & .79 \\
5. Mejora del aprendizaje & 4.04 & .88 \\
6. Motivación & 4.11 & .78 \\
7. Creatividad & 4.14 & .82 \\
8. Escritura & 3.72 & 1.08 \\
9. Integración & 4.03 & .91 \\
10. Mejora de la docencia & 4.31 & .76 \\
\hline
\end{tabular}

Respecto a las actitudes sobre el uso de las diferentes herramientas TIC estas se valoran a partir de un total de 17 ítems (tabla 10). Las herramientas que obtienen una puntuación más alta son el buscador web, con una puntuación de 4.60, el navegador web, con un 4.51 y la plataforma educativa de la Universidad con un 4.46. La puntuación más baja corresponde a los juegos con una puntuación de 2.90 y con un 3.07, las hojas de cálculo.

Tabla 10. Actitudes sobre las diferentes herramientas TIC

\begin{tabular}{lcc}
\hline & Media & $\begin{array}{c}\text { Desviación } \\
\text { estándar }\end{array}$ \\
\hline 1. Procesador de textos & 4.13 & 1.03 \\
2. Bases de datos & 3.81 & 1.05 \\
3. Hojas de cálculo & 3.07 & 1.26 \\
4. Presentaciones & 4.34 & .79 \\
5. Editores de sonido & 3.13 & 1.10 \\
6. Editores de vídeo & 3.43 & 1.11 \\
7. Editores de imagen & 3.45 & 1.11 \\
8. Gestores de correo & 4.15 & .97 \\
9. Navegadores web & 4.51 & .78 \\
10. Buscadores web & 4.60 & .68 \\
11. Editores de blogs/webs & 3.83 & .92 \\
12. Herramientas de trabajo & 4.22 & .89 \\
colaborativas & & \\
13. Plataforma educativa & 4.46 & .81 \\
de la Universidad & & \\
14. Mensajería instantánea & 4.27 & .83 \\
15. Sistemas de & 3.56 & 1.11 \\
videoconferencia & & \\
16. Redes sociales & 4.09 & 1.02 \\
17. Juegos & 2.90 & 1.23 \\
\hline
\end{tabular}

Si analizamos los resultados de manera global, las expectativas y actitudes autopercibidas reciben una puntuación de 3.85 y 4.16 sobre 5, respectivamente. En este caso ocurre, justamente, todo lo contrario, respecto al caso anterior puesto que ambas puntuaciones se encuentran cerca del 4 (sobre 5) (tabla 11).
Tabla 11. Resultados expectativas y actitudes hacia las TIC INCOTIC

\begin{tabular}{ccc}
\hline & Media & $\begin{array}{c}\text { Desviación } \\
\text { estándar }\end{array}$ \\
\hline Expectativas & 3.85 & .53 \\
Actitudes & 4.16 & .59 \\
\hline
\end{tabular}

\subsection{Competencia digital}

Finalmente, en tercer lugar, la autopercepción global de la CD recibe una puntuación de 3.54. Si se desglosa por las distintas alfabetizaciones que la integran se puede ver que: la alfabetización informacional recibe una puntuación de 3.51 ; la alfabetización tecnológica 3.36 ; la alfabetización multimedia de 3.75 ; y la alfabetización comunicacional de 3.62. Siendo así, la multimedia es la que recibe una puntuación más alta y la tecnológica la más baja. Cabe destacar que ambas son las que tienen las desviaciones estándar más elevadas, concretamente de $\quad .67$ y $\quad .80$ respectivamente, implicando que la variedad en las respuestas de los estudiantes puede ser considerable (tabla 12).

Si se analizan los ítems correspondientes a cada una de las alfabetizaciones (tabla 12) se puede observar que el que obtiene una puntuación más alta, concretamente de 4.20, corresponde a la alfabetización comunicativa y, más específicamente, al trabajo colaborativo por parte de los estudiantes. Muy cerca de dicha puntuación se encuentra el correspondiente a la configuración de hardware en los dispositivos, su puntuación es de 4.18. La puntuación más baja es de 2.92 y también corresponde a la alfabetización tecnológica, específicamente se refiere al uso de la ofimática. Muy cerca de dicha puntuación están dos ítems más de la misma alfabetización, concretamente se refieren a la configuración de software y el uso de internet.

Se puede observar que, aunque dentro de la alfabetización tecnológica se encuentre uno de los ítems con una puntuación más alta, también se recogen aquellos menos bien valorados. Este hecho se puede observar en la puntuación global de la alfabetización tecnológica que, como se ha visto previamente, es la que recibe una puntuación más baja. 
Tabla 12. Competencia digital por alfabetizaciones.

\begin{tabular}{|c|c|c|}
\hline & Media & $\begin{array}{l}\text { Desviación } \\
\text { estándar }\end{array}$ \\
\hline Competencia digital & 3.54 & .64 \\
\hline - Informacional & 3.51 & .73 \\
\hline $\begin{array}{l}\text { 1. Reconocer la necesidad } \\
\text { de información }\end{array}$ & 3.79 & .89 \\
\hline 2. Localizar información & 3.45 & 1.22 \\
\hline 3. Evaluar información & 3.62 & 1.06 \\
\hline 4. Organizar información & 3.24 & 1.10 \\
\hline 5. Transformar información & 3.46 & 1.19 \\
\hline - Tecnológica & 3.36 & .87 \\
\hline 6. Configurar hardware & 4.18 & 1.25 \\
\hline 7. Configurar software & 3.00 & 1.27 \\
\hline 8. Usar ofimática & 2.92 & 1.36 \\
\hline 9. Usar Internet & 3.00 & 1.36 \\
\hline 10. Apps específicas & 3.71 & 1.16 \\
\hline - Multimedia & 3.68 & .75 \\
\hline 11. Comprender mensajes & 3.75 & .98 \\
\hline 12. Valorar el estilo & 3.97 & .96 \\
\hline 13. Análisis crítico & 3.79 & .92 \\
\hline 14. Elegir los medios & 2.96 & 1.26 \\
\hline $\begin{array}{l}\text { 15. Producir contenidos } \\
\text { propios }\end{array}$ & 3.98 & 1.34 \\
\hline - Comunicativa & 3.62 & .86 \\
\hline 16. Presentación & 3.23 & 1.21 \\
\hline $\begin{array}{l}\text { 17. Comunicación } \\
\text { asíncrona }\end{array}$ & 3.58 & 1.17 \\
\hline 18. Comunicación síncrona & 4.05 & 1.12 \\
\hline 19. Publicación & 3.10 & 1.33 \\
\hline 20. Trabajo colaborativo & 4.20 & 1.14 \\
\hline
\end{tabular}

\section{DISCUSIÓN}

Los datos recogidos en este estudio muestran que los estudiantes universitarios de educación de primer curso disponen de fácil acceso a la tecnología, pero no hacen mucho uso de esta en el ámbito formal.

La primera pregunta de investigación que nos formulamos, relacionada con el uso que hacen los estudiantes universitarios de las TIC, se ha podido observar que la media de los participantes no hace un uso elevado de ellas. Aún así, es importante tener presente que INCOTIC recoge datos procedentes del contexto formal de los estudiantes.

En esta línea, Guzmán-Simón et al. (2017) destacan que los estudiantes tienen una brecha entre las actividades formales y las informales, siendo las formales en las que menos utilizan las TIC.

En general, se puede observar una elevada desviación estándar en los distintos ítems y, por ello, es difícil delimitar un perfil concreto de estos estudiantes. Los resultados muestran una tendencia hacia el uso de los dispositivos móviles y los ordenadores portátiles. Esta orientación coincide con los informes publicados por AIMC (2018) quienes también apuntan hacia un mayor uso de este tipo de dispositivos.

La segunda pregunta que se plantea en el estudio hace referencia a las expectativas y actitudes que tienen los estudiantes universitarios hacia el uso de las TIC. Se observa que, en general, tienen una percepción positiva hacia su uso. Este resultado coincide con los estudios realizados por Barbosa y Amariles (2019), Prendes, Castañeda y Gutiérrez (2010) y Kivunja (2015).

La tercera pregunta que se plantea en el estudio se refiere al nivel de CD autopercibida por los estudiantes. Entendiendo la CD como el conjunto de las cuatro alfabetizaciones que se consideran en INCOTIC, en este estudio las alfabetizaciones multimedia y comunicativa son las que obtienen una puntuación más elevada. Según Guzmán-Simón et al. (2017) ambas están estrechamente relacionadas con las actividades que realizan los estudiantes en su tiempo de ocio, es decir, en el ámbito informal.

Finalmente, y a nivel general, hay que remarcar que los estudiantes universitarios que han participado en este estudio se otorgan unas puntuaciones altas en los diversos ítems del cuestionario. Como indican Maderick et al. (2015) y Son, Park y Park (2017) los resultados elevados se deben a que la herramienta es de autopercepción y, por tanto, los estudiantes suelen tener una percepción positiva de sus propias habilidades TIC.

\section{CONCLUSIONES}

Este estudio, como ya se ha indicado en la descripción de la herramienta, se ha centrado en la evaluación del nivel de CD autopercibida por parte de los estudiantes universitarios de los grados de educación.

Se considera la CD como una competencia clave para un desempeño adecuado de la profesión docente en el siglo XXI. Ha participado en la investigación una muestra representativa estudiantes (168 de primer curso) de los grados de educación de la Universitat Rovira i Virgili, que han permitido explorar la CD para, a partir de los resultados, continuar mejorando su formación.

La investigación proporciona una visión de la percepción que tienen los estudiantes de primer curso de su nivel de competencia digital y, también, de su uso y expectativa respecto las TIC. El análisis de los datos facilita definir cuáles de las alfabetizaciones de la competencia deben ser abordadas con mayor profundidad en los programas de formación por ser las que tienen un menor grado de percepción. 
Según los resultados, la amplia mayoría de los estudiantes tienen facilidad de acceso a las TIC. De hecho, el $99.4 \%$ dispone de acceso a internet en su domicilio, el $97.6 \%$ disponen de ordenador portátil y el $100 \%$ de teléfonos móviles. Aún así, cabe destacar que no hacen mucho uso de dichos recursos TIC en el ámbito académico.

Con respecto a la CD y las alfabetizaciones que la integran, los estudiantes tienen una autopercepción alta a nivel general y se consideran mejores en la alfabetización multimedia y comunicativa, ya que obtienen una puntuación de 3.68 y 3.62 sobre cinco, respectivamente.

En futuras investigaciones se podría ampliar la muestra con estudiantes procedentes de otras universidades del mismo país o incluso internacionales con la finalidad de contrastar los resultados y ver diferencias y semejanzas entre los diversos contextos. Además, también sería interesante seguir con el análisis de la CD con respecto a las competencias trabajadas en los planes de estudio de la URV.

Finalmente, cabe remarcar que el presente trabajo proporciona unos resultados interesantes respecto al perfil de CD de los estudiantes de primer curso, que pueden contribuir a la mejora de los planes de estudio y el planteamiento de nuevas acciones formativas orientadas al desarrollo y adquisición de la $\mathrm{CD}$ en estudiantes universitarios.

\section{AGRADECIMIENTOS}

La presente investigación agradece la financiación proporcionada mediante el proyecto Simul@b (ref. EDU2013-42223-P) del Ministerio de Economía y Competitividad y por los proyectos de la COMDID1 (2014 ARMIF 0039), COMDID2 (2015 ARMIF 00035) y ACEDIM (2017 ARMIF 00031) del Departament d'Empresa i Coneixement.

\section{REFERENCIAS}

AIMC (2018). Navegantes en la red. Madrid: Asociación Para la Investigación de Medios de Comunicación.

Barbosa Granados, S., y Amariles Jaramillo, M. (2019). Learning Styles and the Use of ICT in University Students within a Competency-Based Training Model. Journal of New Approaches in Educational Research, 8(1), 1-6. doi:10.7821/naer.2019.1.296

Bates, A. W. (2015). Teaching in a digital age. Victoria: BCcampus, BC Open Textbook Project.

Bawden, D. (2008). Origins and concepts of digital literacy. In Digital literacies: Concepts, policies and practices. New York: Peter Lang.

Bullen, M., Morgan, T., y Qayyum, A. (2011). Digital Learners in Higher Education: Rhetoric and reality. International Journal of Excellence in E-Learning, 2(1), 1-13.
Carbonell, X., Chamarro, A., Oberst, U., Rodrigo, B., y Prades, M. (2018). Problematic use of the internet and smartphones in university students: 2006-2017. International journal of environmental research and public health, 15(3), 475.

Carretero, S., Vuorikari, R., y Punie, Y. (2017) DigComp 2.1: The Digital Competence Framework for Citizens (With eight proficiency levels and examples of use). Luxembourg: Publications Office of the European Union.

Comisión Europea. (2018). Recomendación del Consejo de 22 de mayo de 2018 relativa a las competencias clave para el aprendizaje permanente (2018/C 189/01). Diario Oficial de la Unión Europea.

Edmunds, R., Thorpe, M., y Conole, G. (2012). Student attitudes towards and use of ICT in course study, work and social activity: A technology acceptance model approach. British journal of educational technology, 43(1), 71-84.

Erdener, K., y Kandemir, M. A. (2019). Investigation of the reasons for students' attitudes towards the interactive whiteboard use in mathematics classrooms. International Journal of Research in Education and Science (IJRES), 5(1), 331-345.

Erstad, O. (2010). Educating the digital generation. Nordic Journal of Digital Literacy, 5(01), 56-71.

Eshet-Alkalai, Y. (2004). Digital literacy: A conceptual framework for survival skills in the digital era. Journal of Educational Multimedia and Hypermedia, 13(1), 93.

Espuny, C., González, J., Lleixà, M. y Gisbert, M. (2011). Actituds i expectatives de l'ús educatiu de les xarxes socials en els alumnes universitaris. International Journal of Educational Technology in Higher Education (ETHE), 8(1).

Ferrari, A. (2012). Digital competence in practice: An analysis of frameworks. Sevilla: European Commission, Joint Research Centre (JRC).

Gabarda Méndez, V., Rodríguez Martín, A., y Moreno Rodríguez, M. D. (2017). La competencia digital en estudiantes de magisterio. Análisis competencial y percepción personal del futuro maestro. Educatio Siglo XXI, 35, 253-274. doi:10.6018/j/298601

Gallardo-Echenique, E. E., Marqués-Molías, L., Bullen, M., y Strijbos, J. W. (2015). Let's talk about digital learners in the digital era. The International Review of research in open and distributed learning, 16(3).

Gilster, P. (1997). Digital literacy. New York: Wiley Computer.

Gisbert, M., Bullen, M., Punie, Y., Rapetti, E., Galán, F., Vivancos, J., Adell, J., Larraz, V. y Esteve, F. (2016). Key competences. En M. Gisbert y J. González (Eds.), Nous escenaris d'aprenentatge des d'una visió transformadora. Madrid: Wolters Kluwer.

Gisbert, M., González, J., y Esteve, F. (2016). Competencia digital y competencia digital docente: una panorámica sobre el estado de la cuestión. RIITE. Revista Interuniversitaria de Investigación en Tecnología Educativa, 0, 74-83. doi:10.6018/riite/2016/257631.

Gobel, P., y Kano, M. (2013). Student and Teacher use of Technology at the University Level. In IADIS International Conference on Cognition and Exploratory Learning in Digital Age (CELDA 2013).

Gómez, J. (ed.) (2017). UNIVERSITIC 2017. Análisis de las TIC en las Universidades Españolas. Ciudad: Madrid, Editorial: Crue Universidades Españolas.

González-Martínez, J., Esteve, F., Larraz, V., Espuny. C. y Gisbert, M. (2018). INCOTIC 2.0. Una nueva herramienta para la autoevaluación de la competencia digital del alumnado universitario. Profesorado. Revista 
de Currículum y Formación del Profesorado, 22(4), 133-152.

Guzmán-Simón, F., García-Jiménez, E., y López-Cobo, I. (2017). Undergraduate students' perspectives on digital competence and academic literacy in a Spanish University. Computers in Human Behavior, 74, 196204.

Hatlevik, O. E., Throndsen, I., Loi, M., y Gudmundsdottir, G. B. (2018). Students' ICT self-efficacy and computer and information literacy: Determinants and relationships. Computers \& Education, 118, 107-119.

INE (2018). Equipamiento y uso de TIC en los hogares - Año 2018. Madrid: Instituto Nacional de Estadística.

ISTE (2007). NETS-S. NETS for students. The standards for learning, leading, and teaching in the digital age. International Society for Technology in Education.

Kennedy, G., Dalgarno, B., Bennett,... Chang, R. (2009). Educating the net generation. A handbook of findings for practice and policy. Australia: Australian Learning $\mathrm{y}$ Teaching Council.

Kivunja, C. (2015). Teaching Students to Learn and to Work Well with 21st Century Skills: Unpacking the Career and Life Skills Domain of the New Learning Paradigm. International Journal of Higher Education, 4(1), 1-11. doi:10.5430/ijhe.v4n1p1

Kuss, D. J., Kanjo, E., Crook-Rumsey, M., Kibowski, F., Wang, G. Y., y Sumich, A. (2018). Problematic mobile phone use and addiction across generations: The roles of psychopathological symptoms and smartphone use. Journal of Technology in Behavioral Science, 1-9.

Larraz, V. (2013). La competència digital a la universitat (Doctoral dissertation). Universitat d'Andorra, Sant Julià de Lòria.

Liesa Orús, M., Vázquez-Toledo, S., y Lloret-Gazo, J. (2016). Identificación de las fortalezas y debilidades de la competencia digital en el uso de aplicaciones de internet del alumno de primer curso del Grado de Magisterio. Revista Complutense de Educacion, 27(2), 845-862. doi:10.5209/rev_RCED.2016.v27.n2.48409

Maderick, J. A., Zhang, S., Hartley, K., y Marchand, G. (2015). Preservice Teachers and Self-Assessing Digital Competence. Journal of Educational Computing Research, 54(3), 326-351. doi:10.1177/0735633115620432

Mesároš, F., y Mesároš, P. (2010). Digital competencies in process of creating the knowledge company in construction sector. In Proceedings of the 27th ISARC (pp. 544-550).

Mishra, P., y Kereluik, K. (2011). What 21st century learning? A review and a synthesis. En SITE Conference 2011. Nashville, Tennessee, USA.

Moreno, G. C., y Delgado, S. C. (2013). Evaluación de la competencia digital y las actitudes hacia las TIC del alumnado universitario. Revista de Investigación Educativa, 31(2), 536.

Mozilla (2015). Web Literacy: A framework for entry-level web literacy y 21st Century skills. Recuperado desde https://teach.mozilla.org/web-literacy

Nawaz, A., y Kundi, G. M. (2010). From objectivism to social constructivism: The impacts of information and communication technologies (ICTs) on higher education. International Journal of Science and Technology Education Research, 1(2).

OCDE. (2018) The future of education and skills: Education 2030. Directorate for Education and Skills-OECD: France.
Ozdamar-keskin, N., Ozata, F. Z., Banar, K., y Royle, K. (2015). Examining digital literacy competences and learning habits of open and distance learners. Contemporary Educational Technology, 6(1), 74-90.

Prendes, M. P., Castañeda, L., y Gutiérrez, I. (2010). Competencias para el uso de TIC de los futuros maestros. Comunicar, 18(35), 175-182. doi:10.3916/C35-2010-03-11

Prensky, M. (2001). Digital natives, digital immigrants. On the Horizon, 9(5), 1-6.

Roushan, G., Debbie, H., y Biggins, D. (2016). The Kaleidoscope of Voices: An Action Research Approach to Informin Institutional e-Learnin Policy. Electronic Journal of E-Learning, 14(5), 293-300.

Sharp, L. A. (2017). Enhancing Digital Literacy and Learning Among Adults With Blogs. Journal of Adolescent and Adult Literacy, 61(2), 191-202. doi:10.1002/jaal.675

Simonics, I. (2017). Use of ICT equipment by engineer teachers and mentors. In IEEE Global Engineering Education Conference, EDUCON (pp. 527-535).

Son, J.-B., Park, S., y Park, M. (2017). Digital literacy of language learners in two different contexts. JALT CALL Journal, 13(2), 77-96.

Tapscott, D. (1998). Growing up digital: The rise of the net generation. New York: McGraw-Hill.

Vega-Hernández, M. C., Patino-Alonso, M. C., y GalindoVillardón, M. P. (2018). Multivariate characterization of university students using the ICT for learning. Computers \& Education, 121, 124-130.

Vuorikari, R., Punie, Y., Carretero, S., y Van den Brande, L. (2016). DigComp 2.0: The digital competence framework for citizens. Update phase 1: The conceptual reference model. Luxembourg: European Commission. 